\title{
Nonlinear Correlation between Reduced Mass and Computed Activation Energy
}

\author{
Masatoshi Kawashima \\ Laboratory Section, Azuma Co., Ltd. \\ 1-6-13 Tamasaki-Nishi, Ichihara, Chiba 290-0044, Japan \\ E-mail: kawashima@azuma-g.co.jp
}

Correlation between activation energies of the aldol and ene reactions and the reduced masses of the raw materials was revealed by semi-empirical molecular orbital calculations. It was found that there is a singular point where the activation energy changes from decreasing to increasing or to nearly unchanging as the reduced mass increases, and the nonlinear change can be approximated by a quadratic curve. When the coefficient of determination of the correlation was greater than 0.8 , the the reduced mass at the singular point was $40-120$.

\section{Keywords: Reduced Mass | Activation energy | Correlation}

The author has previously reported that it is possible to predict yields in bond formation reactions and dissociation constants in equilibrium reactions from the reduced masses in both cases. ${ }^{1-6}$ And it was clarified that the correlations with the reduced masses are caused by correlations with Gibbs energy change of reaction. ${ }^{7}$

In this study, we shifted our interest to a relationship between activation energy and reduced mass. The relationship between the activation energy and the enthalpy change of reaction has long been known as the Evans-Polanyi principle. However, the relationship between the activation energy and the reduced mass has not been reported so far. ${ }^{8-11}$

First, uncatalyzed aldol reactions of aliphatic aldehydes were chosen to be investigated, and the raw materials with the structures that do not increase the steric hindrance difference between substrates were chosen. ${ }^{12}$ The aldehyde of the nucleophile was set as the enol form. If there is a possibility that isomers may be formed, only the threo form was assumed to be formed as shown in Scheme 1. Combinations of substrates were set out in six ways as shown in Scheme 1. Scheme 1a shows the homo-aldol reaction. In Scheme $1 \mathrm{~b}$ and 1c the nucleophiles are fixed to acetaldehyde and propanal respectively and the carbon number of the aldehyde as an electrophile is changed. In Scheme 1d, 1e and 1f, electrophiles are fixed to acetaldehyde, propanal and butanal respectively and the carbon number of aldehyde as a nucleophile is changed. The transition state was determined from the semiempirical molecular orbital calculation (MOPAC) using SADDLE as the keyword, and after confirming the transition state by TS and FORCE calculation, the activation energy was determined by IRC calculation with IRC $=1 .{ }^{13}$ Figure 1 shows a plot of reduced mass $\left(M_{\mathrm{AB}}=M_{\mathrm{A}} M_{\mathrm{B}} /\left(M_{\mathrm{A}}+M_{\mathrm{B}}\right)\right.$ : where $M_{\mathrm{A}}$ and $M_{\mathrm{B}}$ are molecular weight of molecular $\mathrm{A}$ and $\mathrm{B}$, respectively) versus activation energy $\left(E_{\mathrm{A}}\right)$ for the six types of reactions. In all cases, there are singular points where the activation energy decreases as the reduced mass increases and then gradually increases or remains unchanged.

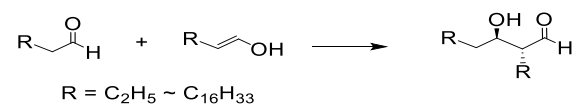

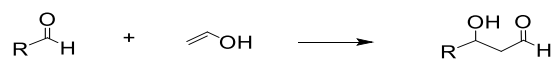

$$
\begin{aligned}
& R^{1}=\mathrm{C}_{2} \mathrm{H}_{5} \sim \mathrm{C}_{79} \mathrm{H}_{159}
\end{aligned}
$$

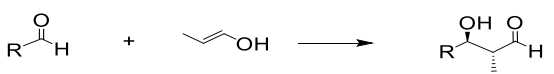

$$
\begin{aligned}
& \mathrm{R}=\mathrm{C}_{2} \mathrm{H}_{5} \sim \mathrm{C}_{49} \mathrm{H}_{99} \\
& {\stackrel{\mathrm{O}}{\mathrm{H}}+\mathrm{R}=\mathrm{R}=\mathrm{C}_{2} \mathrm{H}_{5} \sim \mathrm{C}_{48} \mathrm{H}_{97}}_{\mathrm{R}=}^{\text {OH } \mathrm{O}_{H}}
\end{aligned}
$$

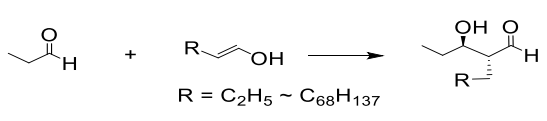

$$
\begin{aligned}
& \sim \mathrm{I}_{\mathrm{H}}+\underset{\mathrm{R}=\mathrm{C}_{2} \mathrm{H}_{5} \sim \mathrm{C}_{48} \mathrm{H}_{97}}{\mathrm{R}} \overbrace{\mathrm{R}-\mathrm{N}^{-}}^{\mathrm{OH}}
\end{aligned}
$$

Scheme 1. Model reactions of aldol reactions for the analysis of correlation between activation energy and reduced mass.

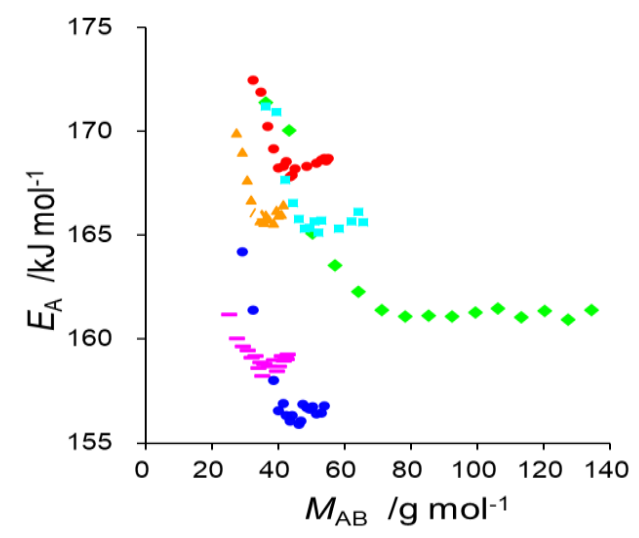

Figure 1. Computed activation energy versus reduced mass plot for six types of aldol reactions. $\downarrow$ : Scheme 1a, -

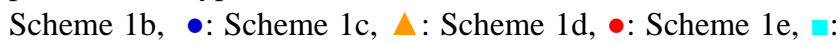
Scheme 1f. 
Next, two types of uncatalyzed carbonyl-ene reactions, shown in Scheme 2, were investigated. ${ }^{14}$ Although there were two reaction points in these reactions, the selectivity was not taken into account, and the calculations were performed as the formalism represented in the scheme. In Scheme $2 a$ and $2 b, 2-$ substituted-1-propenes are used as the olefin compound, and the substituent $\left(\mathrm{R}^{1}\right)$ at the 2-position is increased from methyl group to change the reduced mass. As the enophile, aliphatic aldehydes larger than acetaldehyde are combined in Scheme $2 \mathrm{a}$, and phenylacetaldehydes substituted with a hydrogen atom, a halogen atom, or an aliphatic alkyl group are combined in Scheme $2 b$. In these schemes, the type of carbon chain and the position of substituents are varied so that the steric hindrance and electron density near the reaction site would not change significantly. Calculation results of the activation energies in these reactions shows that there is a singular point at which the activation energy decreases, then increases, or does not change as the reduced mass increases as shown in Figure 2.

$$
\mathrm{R}^{1}=\mathrm{CH}_{3} \sim \mathrm{C}_{17} \mathrm{H}_{35}
$$

Scheme 2. Model reactions of carbonyl-ene reactions for the analysis of correlation between activation energy and reduced mass.

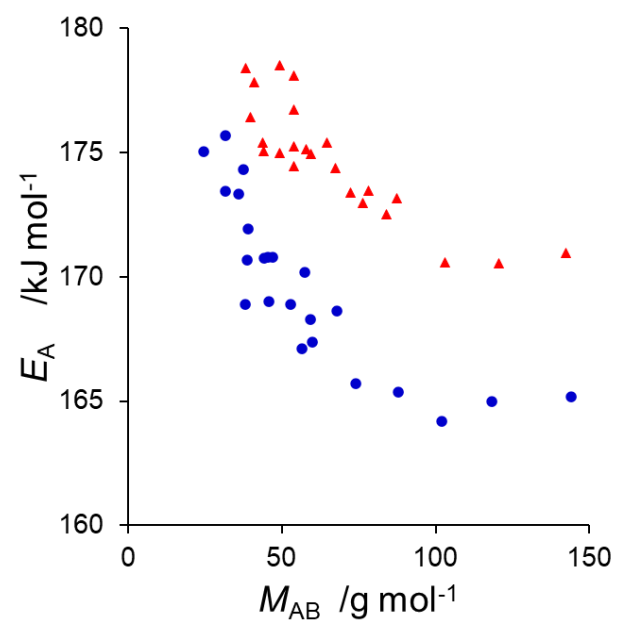

Figure 2. Computed activation energy versus reduced mass plot for two types of carbonyl-ene reactions. •: Scheme 2a, $\Delta$ : Scheme $2 b$.

As the third example of reaction, five types of uncatalyzed ene reactions shown in Scheme 3 were investigated. Even though there were more than two reaction sites in these reactions, the selectivity was not considered and the calculations were performed as the formalism represented in the scheme.

$$
\text { R= } \underset{\substack{\left(1 , 1 ^ { \prime } \text { -biphenyl)-4-yl, } \\ \left(1,1^{\prime}: 4^{\prime}, 1^{\prime \prime}\right.\right. \text {-terphenyl)-4-yl }}}{\mathrm{C}_{18} \mathrm{H}_{37}, \quad \mathrm{R}^{2}=\mathrm{CH}_{3} \sim \mathrm{C}_{19} \mathrm{H}_{39}}
$$

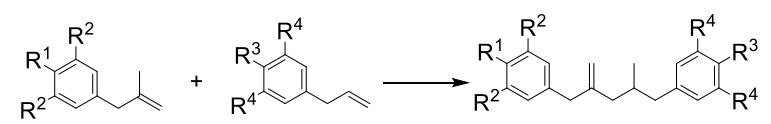

$\mathrm{R}^{1}=\mathrm{H}, \mathrm{NMe}_{2}, \mathrm{NO}_{2}, \mathrm{l}, \quad \mathrm{R}^{3}=\mathrm{H}, \mathrm{NMe}_{2}, \mathrm{NO}_{2}, \mathrm{Cl}, \mathrm{Br}$,

$\mathrm{Ph},\left(1,1^{\prime}\right.$-biphenyl)-4-yl I, Ph, (1,1'-biphenyl)-4-yl

$\mathrm{R}^{2}=\mathrm{H}, \mathrm{F}$

$\mathrm{R}^{4}=\mathrm{H}, \mathrm{F}, \mathrm{I}$

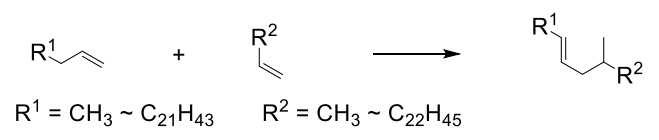

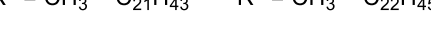

$\mathrm{R}^{1}=\mathrm{CH}_{3} \sim \mathrm{C}_{17} \mathrm{H}_{35} \quad \underset{\mathrm{R}^{2}=\mathrm{CH}_{3} \sim \mathrm{C}_{20} \mathrm{H}_{41}}{\longrightarrow}$

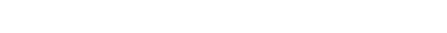

$$
\mathrm{R}^{1}=\mathrm{CH}_{3} \sim \mathrm{C}_{23} \mathrm{H}_{47} \quad \mathrm{R}_{\mathrm{O}}^{2}=\mathrm{CH}_{3} \sim \mathrm{C}_{26} \mathrm{H}_{53}
$$

Scheme 3. Model reactions of ene reactions for the analysis of correlation between activation energy and reduced mass.

In Scheme 3a, alkenes with a hydrogen atom at the allyl position are 2-substituted-1-propenes, and the carbon number is varied as an aliphatic or aromatic hydrocarbon substituent. Counterpart alkenes are 1-alkenes. Scheme $3 \mathrm{~b}$ combines isobutene with a substituted phenyl group as an alkene with a hydrogen atom in the allyl position and 1-propene with a substituted phenyl group as a counter alkene. In Scheme 3c, $3 \mathrm{~d}$, and $3 \mathrm{e}$, alkenes with a hydrogen atom at the allyl position are substituted 1-propenes with an aliphatic substituent at the 3 - position, and counterpart alkenes are 1-alkenes, alkyl vinyl ketones, and alkyl acrylate, respectively. The activation energy was calculated in the same way as before. As a result, similar to the previous reaction example, there seems to be a singular point where the activation energy turns from decreasing to increasing or does not change as the reduced mass increases, as shown in Figure 3 (plot of reduced mass vs. activation energy).

In all cases above, it was found that there is a singularity in which the activation energy turns from decreasing to increasing, or nearly unchanged, as the reduced mass increases. It is interesting to note that the activation energy decreases as the reduced mass increases, even though the possibility of slight steric hindrance due to an increase in the carbon number is not completely eliminated under conditions where the substrate is chosen to be less susceptible to steric hindrance at the reaction site. Although the substituents were placed at the reaction sites so that they would not be affected electronically, calculation results of scheme $3 b$ suggest that they are not affected electronically but by mass. The correlations between the reduced masses and 
activation energies of the 13 reactions in the above scheme 1a3e should be approximated by quadratic functions for the reduced masses below each singular point and by straight lines for the reduced masses above each singular point. However, since it is difficult to increase the amount of data above the singular point due to computational problems, a quadratic curve was used to approximate the whole data before and after the singular point. The coefficients $a, b$, and $c$ of each quadratic function, the reduced mass of the singular point (the reduced mass at the the minimum activation energy of the quadratic function), and the coefficient of determination are summarized in Table 1. In most cases, the coefficient of determination is very high. The low coefficients of determination are probably more influenced by the small coefficient $c\left(\mathrm{R}^{2}=0.57, \mathrm{p}=0.003\right)$ than by the small coefficients $a$ and $b\left(a: \mathrm{R}^{2}=0.27, \mathrm{p}=0.07, b: \mathrm{R}^{2}=0.36, \mathrm{p}=\right.$ $0.03)$. In other words, the larger the activation energy of a reaction, the greater the tendency for the activation energy to decrease when the reduced mass increases. In the equation of the collision theory, the frequency factor part decreases as the reduced mass increases, but the exponential part, including the activation energy, has a larger effect, which means that the reaction rate is highest at the singular point. ${ }^{15}$

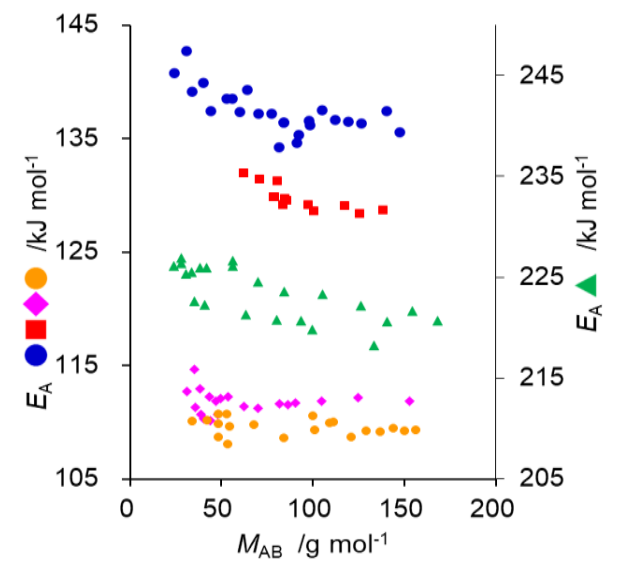

Figure 3. Computed activation energy versus reduced mass plot for five types of carbonyl-ene reactions. •: Scheme (3a), ๘: Scheme (3b), $\Delta$ : Scheme (3c), $\downarrow:$ Scheme (3d), •: Scheme (3e).

Table 1. Coefficients of quadratic approximation formula $\left(E_{\mathrm{A}}\right.$ $\left.=a M_{\mathrm{AB}}^{2}+b M_{\mathrm{AB}}+c\right)$, singular point, and the coefficient of determination.

\begin{tabular}{cccccc}
\hline Scheme & $a$ & $b$ & $c$ & $\begin{array}{c}\text { singular } \\
\text { point }^{2}\end{array}$ & $\mathrm{R}^{2}$ \\
\hline 1a & 0.0043 & -0.7454 & 193 & 87 & 0.96 \\
1b & 0.0179 & -1.3139 & 183 & 37 & 0.91 \\
1c & 0.0246 & -2.3061 & 210 & 47 & 0.96 \\
1d & 0.0470 & -3.4871 & 230 & 37 & 0.96 \\
1e & 0.0233 & -2.1735 & 219 & 47 & 0.94 \\
1f & 0.0185 & -2.0367 & 193 & 55 & 0.96 \\
2a & 0.0014 & -0.3187 & 182 & 114 & 0.86 \\
2b & 0.0006 & -0.1792 & 184 & 149 & 0.78 \\
3a & 0.0007 & -0.1597 & 145 & 114 & 0.68 \\
3b & 0.0011 & -0.2561 & 144 & 116 & 0.82 \\
3c & 0.0003 & -0.1004 & 228 & 167 & 0.60 \\
3d & 0.0002 & -0.0309 & 113 & 77 & 0.043 \\
3e & 0.00002 & -0.0083 & 110 & 208 & 0.073 \\
\hline
\end{tabular}

${ }^{\text {a }}$ reduced mass at the minimum $E_{\mathrm{A}}$.

\section{References and Notes}

1 M. Kawashima, ChemRxiv. 2020, Preprint https://doi.org/10.26434/chemrxiv.12993218.v1

2 M. Kawashima, ChemRxiv. 2020, Preprint. https://doi.org/10.26434/chemrxiv.13350974.v1

3 M. Kawashima, ChemRxiv. 2020, Preprint. https://doi.org/10.26434/chemrxiv.13465718.v1

4 M. Kawashima, ChemRxiv. 2021, Preprint. https://doi.org/10.26434/chemrxiv.13536080.v1

5 M. Kawashima, ChemRxiv. 2021, Preprint https://doi.org/10.26434/chemrxiv.13670737.v1

6 M. Kawashima, ChemRxiv. 2021, Preprint. https://doi.org/10.26434/chemrxiv.14167586.v1.

7 M. Kawashima, ChemRxiv. 2021, Preprint. https://doi.org/10.26434/chemrxiv.14692026.v1

8 M. G. Evans, M. Polanyi, J. Chem. Soc., Faraday Trans. 1936, 32 1340 .

9 S. Zeman, Thermochimica Acta 2002, 384, 137.

10 A. F. Shestakov, Dokl. Phys. Chem. 2003, 393, 339.

11 A. F. Shestakov, Phys. Chem. Chem. Phys. 2005, 7, 2552.

12 An example of activation energy in a catalyzed aldol reaction: J. Yu, A. D. Jensen, L. Wang, C. Li, S. Zhang, Green Chem. 2020, 22, 4222 .

13 Calculations were conducted by MOPAC 6.0 with PM3 using Winmostar (V4, X-Ability Co. Ltd., Tokyo, Japan, 2012) as Gui interface.

14 An example of activation energy in an ene reaction by a DFT study: L. R. Domingo, M. J. Aurell, P. Pérez, Org. Biomol. Chem. 2014, 12,7581 .

15 A. A. Bhalekar, in CLIFFORD ANALYSIS, CLIFFORD ALGEBRAS AND THEIR APPLICATIONS, 2015. CSP Cambridge, UK; I\&S - Florida, USA, 2015, Vol. 4, No. 4, pp. 309340 . 